\title{
Role of IL-17 in the Pathogenesis of Rheumatoid Arthritis
}

\author{
Sarah L. Gaffen, Ph.D. ${ }^{1}$ \\ ${ }^{1}$ Department of Medicine, Division of Rheumatology and Clinical Immunology, University of \\ Pittsburgh, Pittsburgh PA 15261, USA, sig65@pitt.edu.Ph.412-383-8903. Fax.412-383-8864
}

\begin{abstract}
IL-17 (also known as IL-17A) is the signature cytokine of the newly-described "Th17" Thelper cell population, and has been implicated in the pathogenesis of numerous autoimmune diseases including rheumatoid arthritis. IL-17 is the founding member of a new subclass of cytokines that have highly pro-inflammatory properties. Studies in rodents, mammalian cell culture systems as well as clinical settings support a role for IL-17 in promoting rheumatoid arthritis. The history of the discovery of Th17 cells, the potential mechanisms of action of IL-17 in autoimmunity and perspectives for IL-17targeted cytokine therapy are discussed.
\end{abstract}

\section{Introduction}

\section{T helper cells and the new Th17 paradigm}

In the last several years, there has been a paradigm-shift in our understanding of the role of $\mathrm{T}$ cells in autoimmunity, particularly with regards to the CD4+ T helper cell subset (Th cells). $T$ helper cells have long been known as the "masterminds" of the immune system, controlling the activities of other lymphocytes as well as many aspects of the innate immune response. Their depletion in the setting of HIV infection highlights the vital role of CD4+ T cells in coordinating immunity to infectious diseases. Thelper cells accomplish their vital tasks primarily by means of secreting cytokines, protein-based hormones that bind to specific receptors on target cells. Cytokines have pleiotropic activities, which include triggering proliferation, cell death, specific gene expression, cellular migration, etc.

In 1986, a seminal model put forth by Coffman and Mossman postulated that different subpopulations of Th cells could shape immune responses by virtue of differential cytokine production, and two subsets were initially identified that were termed "Th1 and Th2" (Table 1) (1). This model posits that Th1 cells mediate "cellular immunity," characterized by macrophage activation and opsonising antibodies through the actions of the cytokine interferon- $\gamma($ IFN $\gamma$ ). Conversely, Th 2 cells mediate "humoral immunity" characterized by activation of B cells and effector antibodies, which is mediated by interleukin (IL)-4, IL-5 and IL-13 (Figure 1). Central to this model was the concept that naive Th cells are not predetermined to be either Th1 or Th2, but rather that the environment in which they encounter antigen dictates their subsequent fate. In accordance with this idea, it was shown that IL-12, usually derived from antigen-presenting cells (APCs) such as dendritic cells, directs differentiation of naïve CD4+ T cells into the Th1 lineage, whereas IL-4 promotes development to the Th2 lineage. Moreover, differentiation of each Th subset is mutually antagonistic and self-reinforcing by cross-antagonistic signaling pathways mediated by IL-12 and IL-4. For nearly twenty years the Th1/Th2 model provided a valuable framework in which diseases, both infectious and autoimmune, were evaluated. Much research effort was devoted to defining the mechanisms that control Th1/Th2 development, as well as the key cytokines, transcription factors and microbial/autoimmune stimuli involved in this process (reviewed in (2)). 
However, over time it became apparent that there were glaring discrepancies with this model, particularly related to Th1 generation and function (2). The generation of various gene deficient ("knockout") mouse strains revealed puzzling differences between the Th1 effector cytokine IFN $\gamma$ and the Th1 differentiation cytokine IL-12 (Figure 1). IL-12 is a heterodimeric cytokine composed of two subunits, IL-12p40 and IL-12p35. IL-12p40-deficient mice were developed to probe the role of this cytokine in vivo, and IL-12p40-/-mice were found to be resistant to most autoimmune disease models including the rodent model of RA, collagen induced arthritis (CIA) (3). However, IFN $\gamma$-deficient mice and also IFN $\gamma$-receptor deficient mice appeared to be resistant to CIA as well as many other autoimmune disease models; in fact, in some instances these mice showed enhanced sensitivity to disease (3). The basis for this paradox began to become clear when IL-12p35-deficient mice were generated, which appeared to have a highly similar phenotype to the IFN $\gamma$-deficient mice and a less severe phenotype than IL-12p40-/animals. Thus, it was apparent that IL-12p40 plays an IL-12-independent role in immunity. Indeed, IL-12p40 is also component of a new cytokine, IL-23, where it partners with a unique IL-23p19 subunit (Figure 1). Accordingly, IL-12p40-deficient mice are deficient not only in IL-12, but also in IL-23. Direct comparisons of IL-23p19-deficient mice and IL-12p35deficient began to tease apart the biological functions of these two cytokines, and it became clear that many of the autoimmune functions that had previously been ascribed to IL-12 (e.g. in CIA, EAE, IBD), were in fact due to the activities of IL-23 (reviewed in (4)).

Based on these findings, the function of IL-23 became a critical question. In other words, if Th1 cells were not the main effectors of autoimmunity, was there a new, IL-23-dependent T cell subset that had previously been overlooked? Indeed, IL-23 was shown to stimulate production of IL-17 (often referred to as IL-17A) in murine CD4+ T cells (5). IL-17A, although primarily derived from $\mathrm{T}$ cells, was not obviously a Th1 or Th2 cytokine, hinting at the possibility of anew CD4+ T cell lineage involving this cytokine (6). Interestingly, studies in Lyme disease arthritis models demonstrated that a distinct subset of CD4+ T cells produced IL-17A (7), although the connection to IL-23 was not made at that time. Putting this together, two landmark reports showed that an IL-17A-producing CD4+ T cells subset exists that can be induced to differentiate in vitro $(8,9)$. Moreover, induction of this lineage was found to be independent on the classic Th1/Th2 STAT factors STAT4 and STAT6, and development of this so-called "Th17" population could be inhibited by Th1- and Th2-specific cytokines,

IFN $\gamma$ and IL-4 (Table 1). A veritable avalanche of reports followed over the next few years, demonstrating that Th17 cells are indeed a separate lineage (reviewed in (4)). Unexpectedly and in contrast to IL-12, IL-23 was not essential for differentiation of Th17 cells per se, but rather for their pathogenicity in vivo (10). However, in mice IL-23 is nonetheless essential for stable development of Th17 cells, and polymorphisms in the IL-23R gene in humans are linked to susceptibility to Crohn's Disease (11).

Whereas IL-23 is not required for Th17 differentiation, a combination of TGF $\beta$, IL-1, and IL-6 serve in this capacity, and the transcription factor ROR $\gamma \mathrm{t}$ was found to be key for differentiation of these cells (reviewed in (4)). Strikingly, Th17 cells arise in opposition to inducible T regulatory cells (iTregs), in part by virtue of the fact that both lineages depend on TGF $\beta$ for their differentiation $(12,13)$. Thus, in settings of autoimmunity, an altered balance between immunosuppressive Tregs and inflammatory Th17 cells appears to be a major component in disease pathogenesis. Related to this issue, an important and often unappreciated feature of Th cells in vivo are their lineage plasticity. Specifically, differentiation to specific Th1/2/17/Treg phenotypes is not fixed, but rather there is considerable interconversion among these cell types observed in vivo and in vitro $(14,15)$. In particular, Treg and Th17 cells have been shown to interconvert (16), and understanding precisely how and under what circumstances this occurs has important implications in terms of treating autoimmune disease. 
Although IL-17A is predominantly produced by T cells, it is not only the province of the CD4 + subset. CD8+ T cells also produce considerable amounts of IL-17A, as do $\gamma \delta$-T cells, NKT cells and lymphoid tissue inducer (LTi) cells (reviewed in (17)).

\section{IL-17 receptor signaling bridges innate and adaptive immunity}

Although IL-17A is eponymous with the Th17 lineage, these cells also produce IL-17F, IL-21, IL-22, IL-26 (the gene for which is found only in humans), and in some reports TNF $\alpha$, CCL20, and G-CSF (Table 1) (17). Although these are very different cytokines, they all function in a similar manner to promote inflammation, and in some cases can act cooperatively or synergistically (discussed in more detail below). IL-17A and IL-17F are quite homologous $(\sim 55 \%)$ and can form heterodimers (termed IL-17A/F) that appear to be the major isoform of the cytokine in human PBMCs (18). All three cytokine forms bind to a common receptor complex (see below), but exhibit distinct signaling potencies, with IL-17A $>$ IL-17A/F $>$ IL-17F.

IL-17A and IL-17F bind to a multimeric receptor complex composed of at least two subunits, IL-17RA and IL-17RC. These receptors belong to a specific subclass of cytokine receptors with distinct molecular features that distinguish this family from other types of receptors (19). IL-17A and IL-17F signaling through their receptors is unusual compared to typical adaptive Thelper cell cytokines. Rather than activating JAK-STAT pathways, the IL-17-family cytokines activate pro-inflammatory pathways more typical of innate, pro-inflammatory cytokines such as IL-1 or TLR agonists. All these cytokines activate NF- $\mathrm{KB}$, and many also have been shown to induce MAPK signaling and the C/EBP transcription factors. Thus, the net effect of IL-17A signaling is to induce an innate-type inflammatory effector gene expression program that mediates potent inflammation and plays a key role in host defense (reviewed in (20)). Conversely, in conditions of dysregulation, this inflammatory profile can also promote inflammatory pathology in autoimmunity.

Much of our understanding of IL-17A biology in both infection and autoimmunity comes from microarray studies of downstream target genes. Receptors for IL-17 are expressed ubiquitously, but the key responsive cells tend to be non-immune cells such as epithelial cells, mesenchymal cells (myoblasts, fibroblasts and adipocytes, osteoblasts) and keratinocytes. IL-17 stimulates expression of inflammatory genes, including cytokines (IL-6, G-CSF, OSM, IL-32), chemokines (CXCL1, CXCL2, CXCL5, CCL20) and other inflammatory effectors (iNOS, anti-microbial genes, acute phase response genes) (reviewed in (20)). Accordingly, although IL-17A is made by T cells, its downstream signals are similar to those induced by typical "innate" immune receptors such as Toll-like recepor ligands (e.g. LPS) or IL-1 $\beta$.

As indicated above, IL-17A and IL-17F synergize potently with TNF $\alpha$, although the mechanisms underlying this synergy are only partially understood. Thus, IL-17A may act as a "rheostat" for TNF $\alpha$ signaling. A recent study in human synovial fibroblasts (21) compared genes induced by IL-17A versus IL-17F, and found that they promoted similar profiles of gene expression, although IL-17A was quantitatively more active. In the presence of TNF $\alpha$, the patterns of induced genes were highly similar. Interestingly, IL-17A and IL-17F upregulate expression of TNFRII in synoviocytes (21), which may help explain the basis for synergy between these cytokines. IL-17A also cooperates with IL-1 $\beta$ and IL-22, although the synergy is generally not as dramatic as that seen with $\mathrm{TNF} \alpha(22)$. Therefore, Th17-related cytokines act cooperatively to amplify inflammatory cascades, which is beneficial in host defense but deleterious in many autoimmune settings.

\section{IL-17 promotes disease in mouse models of RA}

The pathogenic role of IL-17A in murine models of inflammatory arthritis is unequivocal (23). Elegant work from Erik Lubberts and colleagues has demonstrated a key role for IL-17 
in collage-induced arthritis. This occurs in part due to an altered RANKL:OPG ratio, and is independent of IL-1 and TNF $\alpha$ (reviewed in (24)). IL-17-deficient mice are resistant to CIA (25) as well as spontaneous arthritis in IL-1Ra-deficient mice (26). Blocking IL-17 or its receptor with antibodies reduces disease in mice (reviewed in (24)). Although the mechanisms are not fully elucidated, IL-17 has both direct and indirect bone destructive properties. Th17 cells express much higher levels of RANKL than Th1 or Th2 cells and thus may be very efficient at promoting bone turnover (27). IL-17-induced proteins include matrix metalloproteinases, RANKL and pro-inflammatory effectors such as iNOS that can cumulatively promote bone loss (23). IL-17 signals directly in osteoblasts and synovial fibroblasts to promote inflammation $(21,28)$. Accordingly, IL-17 and other Th17 related cytokines are considered prime targets of anti-cytokine therapy in autoimmune arthritis (24).

The roles of IL-17 and IL-23 on other bone-destructive conditions, however, are complex, and not necessarily pathogenic. For example, the most common form of bone loss in humans is actually due to infectious diseases in the oral cavity that lead to periodontal disease and destruction of the alveolar bone crest of the jaw. Although elevated levels of IL-17 are found in some instances of human periodontal disease (29), in a mouse model IL-17 receptor signaling is highly bone protective through its ability to promote neutrophil expansion and recruitment and hence limit infection (30). IL-23-deficient mice also show abnormalities in maintaining normal bone mass, suggestive of bone-protective properties unrelated to inflammation (31). The extent to which these findings in mice will apply to humans is still unknown, but these observations may raise flags concerning the efficacy of blocking IL-17A or its receptor to treat bone-destructive diseases.

\section{IL-17 in human RA}

There is strong evidence for a role for IL-17 in promoting human RA, although not all studies agree fully. As much as a decade ago, reports began to indicate that high levels of IL-17 and its receptor are found in RA synovial fluid and tissue explants, and IL-17 can promote joint degradation in ex vivo models (32-37). In contrast, there is no convincing data supporting a role for IL-17 in OA. Moreover, IL-17 together with TNF $\alpha$ was found to be predictive for poor outcome in RA (38). IL-17 promotes recruitment of both neutrophils and monocytes by means of inducing various chemokines (37), which can in turn mediate inflammation in RA. However, studies have been reported suggesting that Th1 cells may be more important than Th17 cells (39). The impending use of therapeutics that target IL-17A directly will no doubt shed important light on this issue.

\section{Implications for anti-cytokine therapy}

Blocking TNF $\alpha$ has become a mainstay for treating RA in humans, but is not successful in all situations (reviewed in (40)). TNFa is produced by Th cells but also by macrophages, DCs and other innate immune cells. However, it is also plausible that anti-TNF therapy directly impacts generation of Th17 cells and hence production of IL-17. Consistent with this hypothesis, a recent report examined the consequences of TNF blockade in psoriasis (41). This study of 20 patients reported that $50 \mathrm{mg}$ of etanercept treatment led to improved PASI scores and also to reduced levels of IL-17A and IL-22 in psoriatic lesions. Antibodies that block IL-12p40 (ustekinumab), which hence block both IL-12 and IL-23, were developed based on the premise that Th1 cells promote autoimmune disease. Fortuitously, this subunit is shared with IL-23, and thus this drug presumably limits IL-17A production by blocking Th17 cells as well as Th1 cells. Ustekinumab is quite effective in Crohn's disease and psoriasitic arthritis, although not for multiple sclerosis (42). Similarly, antibodies to the IL-6 receptor (tocilizumab) have shown success for treating RA (43), and IL-6 is both upstream of Th17 cells and a major gene target of IL-17. In contrast, IL-1 appears to be a key driver of Th17 development in human systems, yet Anakinra (a soluble IL-1R antagonist) failed to be an effective therapy for RA even though 
it is highly successful in diseases involving inflammasome defects (44). Although this could be due to its short half-life in vivo, a connection to Th17 cells cannot be ruled out. Antibodies to IL-17A or IL-17RA are presently in Phase I or Phase II clinical trials for a variety of autoimmune diseases, including psoriasis, rheumatoid arthritis, psoriatic arthritis, ankylosing spondylitis and Crohn's Disease (www.clinicaltrials.gov). Early reports of a small psoriasis study indicate that blocking IL-17A is very efficacious, but clearly larger studies will be needed to determine its utility in the clinic (D. Patel, unpublished data). It is likely that, even if blocking IL-17A or its receptor proves efficacious clinically in many patients, it will not be effective in all who take it.

What are the likely safety issues regarding therapeutic blockade of IL-17A? Blocking TNF $\alpha$ therapeutically has proved surprisingly safe, although it can promote development of tuberculosis in patients with latent infections $(44,45)$. Similarly, few major adverse events were reported with the anti-IL-6R Abs (43). Studies using IL-17A-/- or IL-17RA-/- mice have shown an important role for this cytokine system in preventing infections to a long list of microbial pathogens, particularly those that act extracellularly such as gram negative bacteria and the commensal yeast Candida albicans $(46,47)$. An "experiment of nature" has provided valuable insight into the possible side effects of blocking Th17 cells in humans. Specifically, humans with hyper-IgE syndrome (HIES, Job's Syndrome) have recently been shown to have an inherited, dominant-negative defect in the transcription factor STAT3. STAT3 is required for development of Th17 cells due to its role in transducing essential signals from IL-23 and IL-6. Accordingly, HIES patients have a paucity of Th17 cells and IL-17A, but they have normal levels of Th1 cells and IFN $\gamma$ (reviewed in (48)). These patients experience a number of recurrent infectious diseases, including pulmonary infections, staphylococcal abscesses, eczema, and mucocutaneous Candida albicans infections. There are also bone and connective tissue disorders related to other functions of STAT3 (reviewed in (49)). Thus, blocking IL-17A may prove problematic in terms of enhancing susceptibility to common infections.

\section{Conclusions and perspectives}

The past few years have been a watershed in our understanding of the T cell-mediated pathogenesis of autoimmune disease. The impact of the revision of the Th1/Th2 paradigm to include Th17 cells cannot be overstated. Nonetheless, it is striking that many of the anticytokine therapies that have been developed to target Th1 cells in fact seem to act through the Th17 lineage instead. It should be noted that, although the Th1 and Th17 cell types are often considered separately, in fact there is much crosstalk between them (reviewed in \{Korn, 2009 \#2199; O'Quinn, 2008\#2216\}). Understanding how Th17 cells and their downstream cytokines act at a fundamental level is likely to reveal new strategies for treating RA and other autoimmune conditions. Conversely, defining the potential side effects of blocking Th17 cells in animal models as well as rare human populations with genetic defects in these cells is critical for predicting and being prepared for potential complications associated with this approach.

\section{Acknowledgments}

SLG is supported by research grants from the Alliance for Lupus Research, Amgen and the NIH (AR054389, DE019424). SLG has received travel reimbursement, honoraria and a research grant from Amgen. I thank D. Patel (Novartis) for sharing unpublished information.

\section{References}

1. Mosmann TR, Cherwinski H, Bond MW, et al. Two types of murine helper T cell clone. I. Definition according to profiles of lymphokine activities and secreted proteins. J Immunol 1986;136:2348-2357. [PubMed: 2419430] 
2. Steinman L. A brief history of $\mathrm{T}(\mathrm{H}) 17$, the first major revision in the $\mathrm{T}(\mathrm{H}) 1 / \mathrm{T}(\mathrm{H}) 2$ hypothesis of $\mathrm{T}$ cell-mediated tissue damage. Nature Med 2007;13:139-145. [PubMed: 17290272] * This is an excellent review article detailing the historical perspective by which discrepancies in the Th1/Th2 paradigm were viewed and the significance of the Th17 cell discovery.

3. Murphy CA, Langrish CL, Chen Y, et al. Divergent pro- and antiinflammatory roles for IL-23 and IL-12 in joint autoimmune inflammation. The Journal of experimental medicine 2003;198:1951-1957. [PubMed: 14662908]

4. McGeachy MJ, Cua DJ. The link between IL-23 and Th17 cell-mediated immune pathologies. Semin Immunol 2007;19:372-376. [PubMed: 18319054]

5. Aggarwal S, Ghilardi N, Xie MH, et al. Interleukin 23 promotes a distinct CD4 T cell activation state characterized by the production of interleukin 17 . The Journal of biological chemistry 2002;3:19101914. [PubMed: 12417590]

6. Aarvak T, Chabaud M, Miossec P, Natvig JB. IL-17 is produced by some proinflammatory Th1/Th0 cells but not by Th2 cells. J Immunol 1999;162:1246-1251. [PubMed: 9973376]

7. Infante-Duarte C, Horton HF, Byrne MC, Kamradt T. Microbial lipopeptides induce the production of IL-17 in Th cells. J Immunol 2000;165:6107-6115. [PubMed: 11086043]

8. Park H, Li Z, Yang XO, et al. A distinct lineage of CD4 T cells regulates tissue inflammation by producing interleukin 17. Nature immunology 2005;6:1133-1141. [PubMed: 16200068]

9. Harrington LE, Hatton RD, Mangan PR, et al. Interleukin 17-producing CD4+ effector T cells develop via a lineage distinct from the T helper type 1 and 2 lineages. Nature immunology 2005;6:1123-1132. [PubMed: 16200070]

10. McGeachy MJ, Bak-Jensen KS, Chen Y, et al. TGF-beta and IL-6 drive the production of IL-17 and IL-10 by T cells and restrain T(H)-17 cell-mediated pathology. Nature immunology 2007;8:13901397. [PubMed: 17994024]

11. Duerr RH, Taylor KD, Brant SR, et al. A Genome-Wide Association Study Identifies IL23R as an Inflammatory Bowel Disease Gene. Science 2006;314:1461-1463. [PubMed: 17068223]

12. Bettelli E, Carrier Y, Gao W, et al. Reciprocal developmental pathways for the generation of pathogenic effector $\mathrm{T}(\mathrm{H}) 17$ and regulatory T cells. Nature 2006;441:235-238. [PubMed: 16648838]

13. Mangan PR, Harrington LE, O'Quinn DB, et al. Transforming growth factor-beta induces development of the T(H)17 lineage. Nature 2006;441:231-234. [PubMed: 16648837]

14. Wei G, Wei L, Zhu J, et al. Global mapping of H3K4me3 and H3K27me3 reveals specificity and plasticity in lineage fate determination of differentiating CD4+ T cells. Immunity 2009;30:155-167. [PubMed: 19144320]

15. Lee YK, Turner H, Maynard CL, et al. Late developmental plasticity in the T helper 17 lineage. Immunity 2009;30:92-107. [PubMed: 19119024]

16. Lohr J, Knoechel B, Caretto D, Abbas AK. Balance of Th1 and Th17 effector and peripheral regulatory T cells. Microbes Infect. 2009

17. Korn T, Bettelli E, Oukka M, Kuchroo VK. IL-17 and Th17 Cells. Annual review of immunology 2009;27:485-518.

18. Wright JF, Guo Y, Quazi A, et al. Identification of an Interleukin 17F/17A Heterodimer in Activated Human CD4+ T Cells. The Journal of biological chemistry 2007;282:13447-13455. [PubMed: 17355969]

19. Gaffen SL. Structure and signalling in the IL-17 receptor superfamily. Nat Rev Immunol. 2009 in press.

20. Shen F, Gaffen SL. Structure-function relationships in the IL-17 receptor: Implications for signal transduction and therapy. Cytokine 2008;41:92-104. [PubMed: 18178098]

21. Zrioual S, Ecochard R, Tournadre A, et al. Genome-wide comparison between IL-17A- and IL-17Finduced effects in human rheumatoid arthritis synoviocytes. J Immunol 2009;182:3112-3120. [PubMed: 19234208] * This paper elegantly compares genes induced by IL-17A versus IL-17F in human RA synovial fibroblasts, and reveals important insights into mechanisms of synergy with $\mathrm{TNF} \alpha$

22. Liang SC, Tan XY, Luxenberg DP, et al. Interleukin (IL)-22 and IL-17 are coexpressed by Th17 cells and cooperatively enhance expression of antimicrobial peptides. The Journal of experimental medicine 2006;203:2271-2279. [PubMed: 16982811] 
23. Toh ML, Miossec P. The role of T cells in rheumatoid arthritis: new subsets and new targets. Curr Opin Rheumatol 2007;19:284-288. [PubMed: 17414957]

24. Lubberts E. IL-17/Th17 targeting: on the road to prevent chronic destructive arthritis? Cytokine 2008;41:84-91. [PubMed: 18039580] ** This review summarizes the role of IL-17 and its receptor in RA, and provides important clinical perspectives into its use in therapy

25. Nakae S, Nambu A, Sudo K, Iwakura Y. Suppression of immune induction of collagen-induced arthritis in IL-17-deficient mice. J Immunol 2003;171:6173-6177. [PubMed: 14634133]

26. Nakae S, Saijo S, Horai R, et al. IL-17 production from activated T cells is required for the spontaneous development of destructive arthritis in mice deficient in IL-1 receptor antagonist. Proc Natl Acad Sci U S A 2003;100:5986-5990. [PubMed: 12721360]

27. Sato K, Suematsu A, Okamoto K, et al. Th17 functions as an osteoclastogenic helper T cell subset that links $\mathrm{T}$ cell activation and bone destruction. The Journal of experimental medicine 2006;203:2673-2682. [PubMed: 17088434]

28. Shen F, Ruddy MJ, Plamondon P, Gaffen SL. Cytokines link osteoblasts and inflammation: microarray analysis of interleukin-17- and TNF-alpha-induced genes in bone cells. J Leukoc Biol 2005;77:388-399. [PubMed: 15591425]

29. Gaffen SL, Hajishengallis G. A New Inflammatory Cytokine on the Block: Rethinking periodontal disease and the Th1/Th2 paradigm in the context of Th17 cells and IL-17. J Dent Res 2008;87:817828. [PubMed: 18719207]

30. Yu J, Ruddy M, Wong G, et al. An essential role for IL-17 in preventing pathogen-initiated bone destruction: Recruitment of neutrophils to inflamed bone requires IL-17 receptor-dependent signals. Blood 2007;109:3794-3802. [PubMed: 17202320]

31. Quinn JM, Sims NA, Saleh H, et al. IL-23 inhibits osteoclastogenesis indirectly through lymphocytes and is required for the maintenance of bone mass in mice. J Immunol 2008;181:5720-5729. [PubMed: 18832731]

32. Kotake S, Udagawa N, Takahashi N, et al. IL-17 in synovial fluids from patients with rheumatoid arthritis is a potent stimulator of osteoclastogenesis. J Clin Invest 1999;103:1345-1352. [PubMed: 10225978]

33. Ziolkowska M, Koc A, Luszczukiewicz G, et al. High levels of IL-17 in rheumatoid arthritis patients: IL-15 triggers in vitro IL-17 production via cyclosporin A-sensitive mechanism. J Immunol 2000;164:2832-2838. [PubMed: 10679127]

34. Honorati MC, Meliconi R, Pulsatelli L, et al. High in vivo expression of interleukin-17 receptor in synovial endothelial cells and chondrocytes from arthritis patients. Rheumatology (Oxford) 2001;40:522-527. [PubMed: 11371660]

35. Chabaud M, Fossiez F, Taupin JL, Miossec P. Enhancing effect of IL-17 on IL-1-induced IL-6 and leukemia inhibitory factor production by rheumatoid arthritis synoviocytes and its regulation by Th2 cytokines. J Immunol 1998;161:409-414. [PubMed: 9647250]

36. Cai L, Yin JP, Starovasnik MA, et al. Pathways by which interleukin 17 induces articular cartilage breakdown in vitro and in vivo. Cytokine 2001;16:10-21. [PubMed: 11669582]

37. Shahrara S, Pickens SR, Dorfleutner A, Pope RM. IL-17 induces monocyte migration in rheumatoid arthritis. J Immunol 2009;182:3884-3891. [PubMed: 19265168]

38. Kirkham BW, Lassere MN, Edmonds JP, et al. Synovial membrane cytokine expression is predictive of joint damage progression in rheumatoid arthritis: a two-year prospective study (the DAMAGE study cohort). Arthritis and rheumatism 2006;54:1122-1131. [PubMed: 16572447]

39. Yamada H, Nakashima Y, Okazaki K, et al. Th1 but not Th17 cells predominate in the joints of patients with rheumatoid arthritis. Annals of the rheumatic diseases 2008;67:1299-1304. [PubMed: 18063670]

40. Scheinecker C, Redlich L, Smolen J. Cytokines as thereapeutic targets: Advances and limitations. Immunity 2008;28:440-444. [PubMed: 18400186]

41. Zaba LC, Cardinale I, Gilleaudeau P, et al. Amelioration of epidermal hyperplasia by TNF inhibition is associated with reduced Th17 responses. The Journal of experimental medicine 2007;204:31833194. [PubMed: 18039949] ** This paper shows that the success of TNF $\alpha$ blockade may in part be due to suppressive effects on Th17 activity 
42. Reich K, Yasothan U, Kirkpatrick P. Ustekinumab. Nat Rev Drug Discov 2009;8:355-356. [PubMed: 19404310]

43. Smolen JS, Beaulieu A, Rubbert-Roth A, et al. Effect of interleukin-6 receptor inhibition with tocilizumab in patients with rheumatoid arthritis (OPTION study): a double-blind, placebocontrolled, randomised trial. Lancet 2008;371:987-997. [PubMed: 18358926]

44. Mclnnes IB, Schett G. Cytokines in the pathogenesis of rheumatoid arthritis. Nat Rev Immunol 2007;7:429-442. [PubMed: 17525752]

45. Strangfeld A, Listing J. Infection and musculoskeletal conditions: Bacterial and opportunistic infections during anti-TNF therapy. Best Pract Res Clin Rheumatol 2006;20:1181-1195. [PubMed: 17127203]

46. O'Quinn D, Palmer M, Lee Y, Weaver C. Emergence of the Th17 pathway and its role in host defense. Adv Immunol 2008;99:115-163. [PubMed: 19117534]

47. Conti H, Shen F, Nayyar N, et al. Th17 cells and IL-17 receptor signaling are essential for mucosal host defense against oral candidiasis. The Journal of experimental medicine 2009;206:299-311. [PubMed: 19204111]

48. Fischer A. Connecting STAT3, Th17 and human mucosal immunity. Immunol Cell Biol 2008;86:549-551. [PubMed: 18645579] * This review summarizes the discovery of Th17-deficient humans with Hyper-IgE syndrome, which may provide important insight into the consequences of IL-17 blockade for therapy

49. Freeman AF, Holland SM. The hyper-IgE syndromes. Immunology and allergy clinics of North America 2008;28:277-291. viii. [PubMed: 18424333] 
Old model

Current model

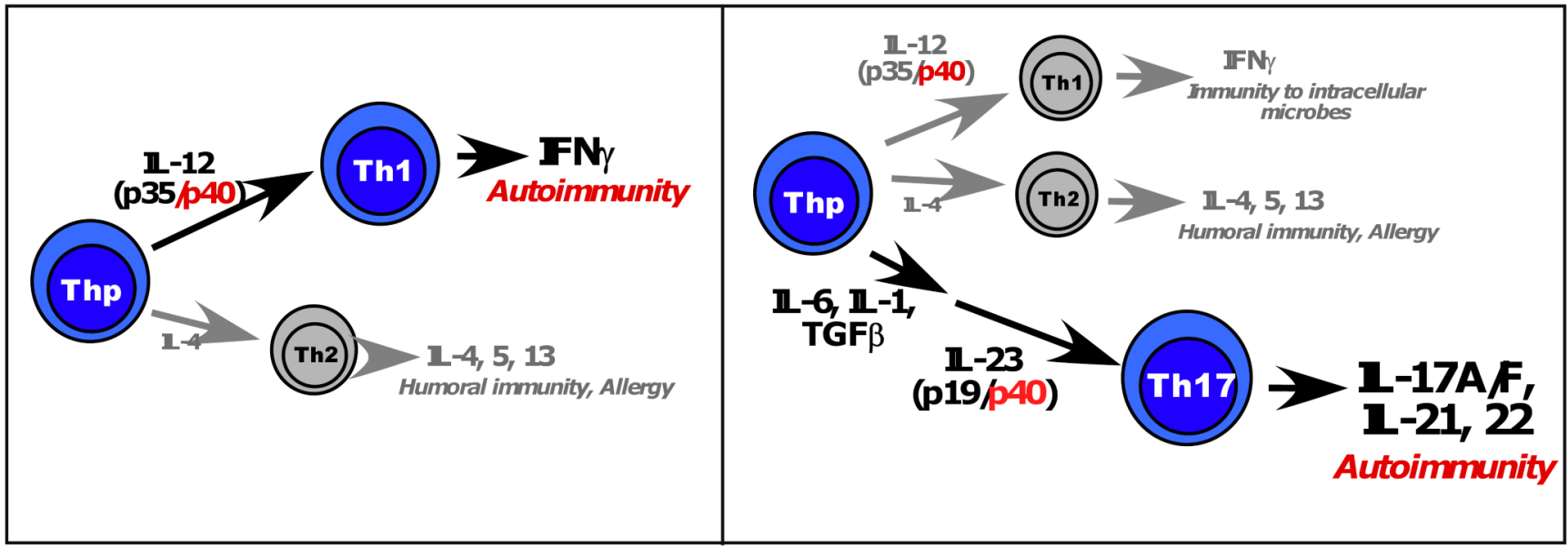

Figure 1. Old versus new models of Th cell development

In the original paradigm of CD4+ T cell differentiation, most CD4+ T helper cells could be classified into Th1 cells (typified by production of IFN $\gamma$ ) or Th2 cells (typified by production of IL-4, IL-5 and IL-13). Th1 cells were considered to promote host defense against most infectious microbes (both intracellular and extracellular), whereas $\mathrm{Th} 2$ cells were thought to act primarily against large Helminth worms. Th1 cells were also considered to be the major driver of autoimmune pathology in RA as well as other autoimmune diseases such as psoriasis, multiple sclerosis and Crohn's Disease. The heterodimeric cytokine IL-12, composed of the IL-12p40 and IL-12p35 subunits, was shown to be critical for development of Th1 cells, and hence efforts to block IL-12 with antibodies against the IL-12p40 subunit were pursued. In 2005, the discovery of the Th17 subset revised this model, which was partly revealed based on a new understanding of the shared role of the IL-12p40 subunit in the cytokine IL-23. Th17 cells are driven to differentiation by a combination of IL-1, IL-6 and TGF $\beta$, and IL-23 (composed of the IL-12p40 and the IL-23p19 subunits) serves to expand and stabilize this lineage. 
Table 1

T helper subsets and their major functions

\begin{tabular}{|l|l|l|l|l|}
\hline & Th1 & Th2 & Th17 & iTreg \\
\hline Major cytokines & IFN $\gamma$ & IL-4, IL-5, IL-13 & $\begin{array}{l}\text { IL-17A, IL-17F, } \\
\text { IL-21, IL-22, IL-26 } \\
\text { (humans), TNF }, \\
\text { CCL20 }\end{array}$ & IL-10, TGF \\
\hline Main Antimicrobial activity & Intracellular microbes & Helminths & Extracellular microbes & $\begin{array}{l}\text { Limit immune- } \\
\text { mediated } \\
\text { damage, } \\
\text { prevent } \\
\text { autoimmunity }\end{array}$ \\
\hline Inductive cytokines & IL-12 & IL-4 & IL-1, IL-6, TGF $\beta$ & TGF $\beta$, IL-2 \\
\hline Inductive transcription factors & STAT1, Tbet & STAT6, GATA3 & STAT3, ROR $\gamma \mathrm{t}$ & STAT5, Foxp3 \\
\hline
\end{tabular}

